\title{
Modeling Spectral Changes to Visualize Embedded Volume Structures for Medical Image Data
}

\author{
H.J. Noordmans ${ }^{1}$, H.T.M. van der Voort $^{2}$, and M.A. Viergever ${ }^{1}$ \\ 1 Image Sciences Institute E.01.334, P.O. Box 85500 \\ University Hospital Utrecht, 3508 GA Utrecht, The Netherlands \\ herke@isi.uu.nl \\ http://www.isi.uu.nl/People/Herke/herke.html \\ 2 Scientific Volume Imaging BV \\ Alexanderlaan 14, 1213 XS Hilversum, The Netherlands \\ info@svi.nl \\ http://www.svi.nl/
}

\begin{abstract}
When a volume structure is embedded in another volume structure, it is difficult to see the inner structure without destroying the view on the outer structure. We present a solution by letting the volume structures scatter light with a different wavelengths. The outer volume structure only absorbs light scattered by the structure itself and does not absorb the light scattered by the inner structure, by which the inner structure is visible without destroying the view on the outer structure. Examples are shown of vascular imaging and CT imaging.
\end{abstract}

\section{Introduction}

The increasing number of image modalities, their increasing quality and the progress in image segmentation algorithms increase the number of volume structures one wants to combine in a volume rendering. This can be done by giving each structure a distinct color and opacity. However it becomes more complicated to show a structure which is embedded in another volume structure. One can think of seeing the organs inside the skin of the surrounding abdomen for laprascopy or to see the veins surrounding the brain in order to avoid them in neurosurgery. It is possible to cut a virtual hole in the outer structure[1] or to decrease its opacity, but a disadvantage is loosing surface information of a part or the total outer structure. If one still wants to see surface detail, for example for navigation, the visualization problem is more difficult. A simple option is to render the two structures separately and combine them at display. Possibilities are taking the square root of their squared intensities[2], putting them in a separate RGB channel[2], or averaging the RGB colors. The result may be satisfying, but the question remains whether there exists a less ambiguous and less ad-hoc way of visualizing an embedded volume structure. 


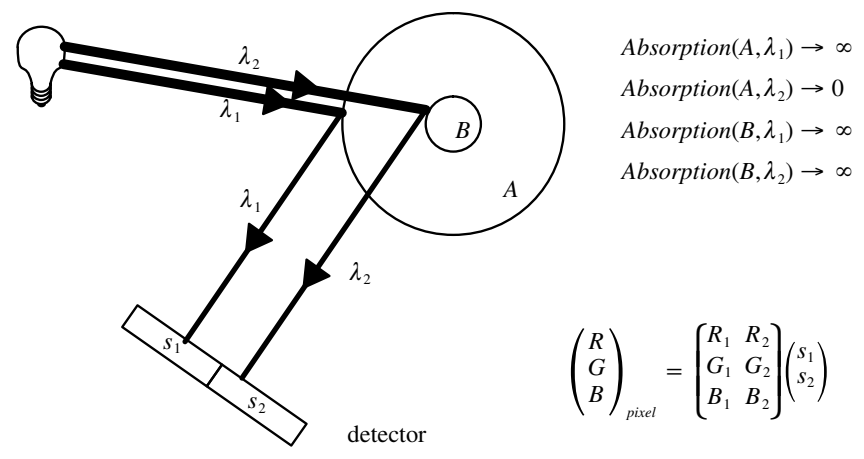

Fig. 1. Schematic layout spectral volume rendering.

\section{Spectral Approach}

In our search for such a 'ideal' method, we started by looking at nature. In the real world, a light source emits light that illuminates an object. The light is absorbed, reflected and scattered by the object before it reaches our eye. The light has a spectrum (light has a color not an object!) which changes in the absorption, reflecting, scattering process. This 'changed' spectrum is seen by our eyes. The human eye can be considered as a detector which, by its cones, converts the spectrum into a kind of RGB signal. In our volume renderer we simulate the same principle (Simulated Fluorescence Process[3]). We simulate a light source emitting an illumination spectrum. We assume that a voxel contains an amount of material with specific scatter and absorbing properties. The value of the voxel determines the amount, a label the type of material. The illumination spectrum excites the material which on its turn emits a part of it. A part of the light is absorbed and a part passes the voxel to illuminate other voxels. The emitted spectrum of the materials is seen by a detector which contains a kind of spectrum analyzer. The result is a sequence of numbers which is mapped by a matrix to an RGB value.

Returning to our problem, to visualize object B inside object A (Fig. 1), we assume that the illumination spectrum consists of two main wavelength bands, characterized by $\lambda_{1}$ and $\lambda_{2}$. Object A has material with strong absorption bands around $\lambda_{1}$ but with almost no absorption around $\lambda_{2}$. Object $\mathrm{B}$ absorbs spectral bands both around $\lambda_{1}$ and $\lambda_{2}$. Object A only scatters the spectral band around $\lambda_{1}$, while object B only scatters the spectral band around $\lambda_{2}$. The detector sees both wavelength bands and converts them to an RGB value. The result is that we see surface detail of object A while seeing object B through it. 

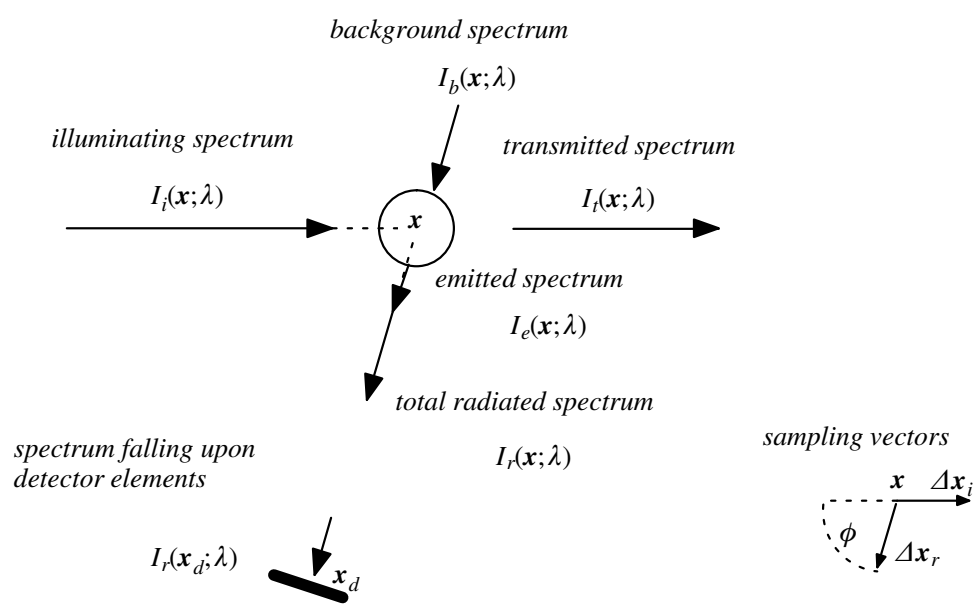

Fig. 2. Spectra involved in light/matter interaction process.

\subsection{Theoretical Background}

Although the spectral volume renderer can be reduced to a more common $\mathrm{RGB} \alpha$ volume renderer [4], [5], [6], the main difference is the more physically realistic basis which underlies our renderer. Whereas in an $\mathrm{RGB} \alpha$ renderer rays are traced through an $\mathrm{RGB} \alpha$ volume (where each voxel is described by a RGB triplet and opacity $\alpha$ ), we simulate how light from a light source interacts with materials inside a voxel. Each segmented object or image modality is assumed to consist of one type of material $m$. By letting the material types have different absorbing and scattering properties, objects have a different opacity and color.

There are many physical ways how light can interact with a material. The material can reflect it perfectly (e.g. metal sphere), absorb some of the incident energy (e.g. convert it into heat), scatter it elastically (no energy loss, Rayleigh scattering), or inelastically (energy loss, e.g. phosphoresce, fluorescence). This process is angle and wavelength dependent. As we are not trying to simulate nature, but only want to improve volume rendering such that we have a better understanding of the volume data, we omit the (in our eyes disturbing) angular dependence. We also restrict ourselves to low-albedo scattering, i.e. light is only scattered once and not multiple times, as we want sharp looking shadows and not diffuse ones. The advantage of low-albedo scattering is that the scattering process can be divided into a illumination and radiation phase[7].

Putting it mathematically, the spectral changes which occur in the illumination phase can be written as (Fig. 2):

$$
\begin{aligned}
I_{t}(\vec{x}, \lambda) & =I_{i}(\vec{x}, \lambda) \cdot\left(1-\sum_{m=1}^{M} \rho(\vec{x}, m) A(m, \lambda)\right), \\
I_{i}\left(\vec{x}+\Delta \overrightarrow{x_{i}}, \lambda\right) & =I_{t}(\vec{x}, \lambda),
\end{aligned}
$$


where $\rho(\vec{x}, m)$ describes the density of material type $m$ at voxel position $\vec{x}$, and $A(m, \lambda)$ the spectral absorption of material type $m$. The second part of Eq. 1 describes how the transmitted spectrum $I_{t}(\vec{x}, \lambda)$ acts as the illumination spectrum $I_{i}(\vec{x}, \lambda)$ for the next sampling position.

In the radiation phase, we have similar equations:

$$
\begin{aligned}
I_{r}(\vec{x}, \lambda) & =I_{e}(\vec{x}, \lambda)+I_{b}(\vec{x}, \lambda) \cdot\left(1-\sum_{m=1}^{M} \rho(\vec{x}, m) A(m, \lambda)\right), \\
I_{b}\left(\vec{x}+\Delta \overrightarrow{x_{r}}, \lambda\right) & =I_{r}(\vec{x}, \lambda) .
\end{aligned}
$$

The difference is that materials now add energy during the ray tracing process. Note that for an efficient implementation the equation should be rewritten in a front to back manner in order to use adaptive ray termination[4].

To incorporate different kinds of scattering processes the emitted spectrum is also a function of the incident spectrum:

$$
I_{e}(\vec{x}, \lambda)=\sum_{m=1}^{M} \rho(\vec{x}, m) E\left(m, \lambda, I_{i}\right) .
$$

The final spectrum emerging from the data volume falls upon a set of detectors which converts the spectra into an RGB signal:

$$
\left(\begin{array}{c}
R \\
G \\
B
\end{array}\right)=\left(\begin{array}{ll}
\int_{\lambda=-\infty}^{\infty} I_{r}\left(x_{d}\right) s_{R}(\lambda) d \lambda \\
\int_{\lambda=-\infty}^{\infty} I_{r}\left(x_{d}\right) s_{G}(\lambda) d \lambda \\
\int_{\lambda=-\infty}^{\infty} I_{r}\left(x_{d}\right) s_{B}(\lambda) d \lambda
\end{array}\right),
$$

where $s_{R}(\lambda), s_{G}(\lambda)$, and $s_{B}(\lambda)$ are the sensitive spectra of respectively the red, green and blue element. This RGB value defines the final color of the view pixel. Note that this is a major difference of our method compared to other methods: that the color of an object is not fully determined by the material itself, but also by the illumination source and the light detector.

Although Eqs 1 to 3 allow you to simulate the light interacting with real materials, we chose for 'ideal' materials which scatter only a specific band of the spectrum in order to speed up the rendering process. To have all degrees of freedom, each material should be associated with one spectral band $\lambda_{m}$.

To get the behavior to selectively let pass light by embedded objects (Fig. 1), but not change the color of objects, we looked how materials could influence the volume rendering process. Looking at Fig. 2, we see that there are three instances at which materials influence the light spectrum: (a) Materials may absorb some of the spectral components during illumination, (b) materials may change the spectrum in the scattering process itself, and (c) materials may absorb some of the spectral components of light emitted by other materials. We investigated three types of scattering processes:

- Achromatic scattering. As each wavelength is scattered with the same efficiency, the emitted photon has the same wavelength as the incident photon. An example is a perfectly reflecting sphere. 


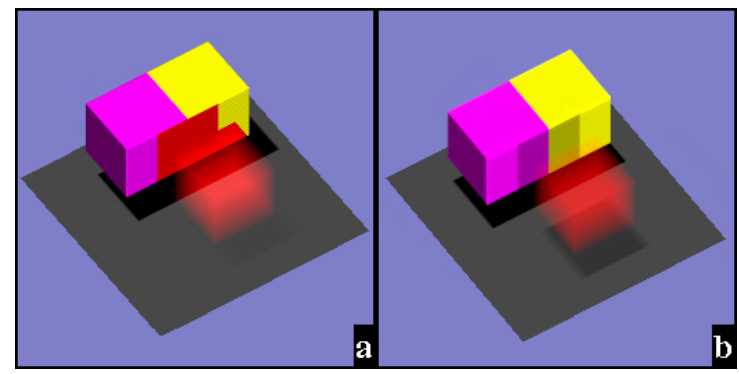

Fig. 3. Neutral transmittance. a Violation, the red block with chromatic absorption makes the transmitted light red. The purple and yellow block can no longer be distinguished in the shadow; $\mathbf{b}$ Agreement, the red block with achromatic absorption does not change the color of the transmitted light, the purple and yellow block can still be distinguished in the shadow. Note that there are two light sources: one from above, and one from the front.

- Elastically scattering. The emitted photon has the same wavelength as the incident photon, but the materials do not equally scatter all wavelengths.

- Inelastically scattering. As energy is lost in the inelastic scattering process, the emitted photon has a longer wavelength that the incident photon. Also, not all wavelengths are scattered equally.

When considering absorbing characteristics, we distinguish two types:

- Achromatic absorption. All spectral components are absorbed to the same extend. The overall absorption may vary from material to material.

- Chromatic absorption. One spectral component may be more absorbed than another. The absorption is wavelength dependent.

We combine the three scattering processes and the two absorption types into six material models. We examine them one by one on the following requirements: (a) potential of object discrimination by color, (b) visibility of interactive tools, (c) ability to render unsegmented images, and (d) neutral transmittance. Objects have a neutral transmittance when they do not influence the color of another object seen or illuminated through them (Fig. 3), permitting clear object identification. In this examination, we assume a white light source, i.e. a source radiating a uniform spectrum.

1. Achromatic scattering with achromatic absorption. For achromatic scattering, the shape of the light spectrum does not change in the scattering process. As materials differ only in their scattering efficiency and as the absorption is achromatic, discrimination among different volume objects is only possible with different transparencies, but not with colors.

2. Achromatic scattering with chromatic absorption. The chromatic absorption inevitably influences the illuminating light spectrum, with the effect 
that semi-transparent objects change the color of other objects (Fig. 3). Also, within one material there is a gradual change in color of light passing through. Both effects are a violation of the principle of neutral transmittance.

3. Elastic scattering with achromatic absorption. In this model the absorption spectra of the materials are constant. Only the scattering process influences the shape of the radiated spectrum. The perceived color is determined only by the volume object and there is no color interference by other volume objects. The principle of neutral transmittance is satisfied.

4. Elastic scattering with chromatic absorption. The light spectrum scattered by a material is influenced by the scattering efficiency of the material itself, and by all absorption spectra of materials the light spectrum has traversed. Similarly to model 2 , the chromatic absorption violates the principle of neutral transmittance. The violation does not occur under specific conditions: If the spectral range of the emitted spectra is limited, and if the media do not change the shape of these spectra. Then the media can attenuate these spectra without changing their shape, thereby satisfying the principle of neutral transmittance. The advantage of wavelength dependent media is that media can be chosen in such a way that they selectively pass light scattered by other materials. An object of one material might absorb light scattered by the material itself, but passes light scattered by materials of an inside object (Fig. 1). This is particularly useful to bring to the front otherwise occluded interactive tools.

5. Inelastically scattering with achromatic absorption. As the absorption is achromatic, materials cannot selectively suppress light scattered by other materials. The visualization possibilities are similar to those of model 3 .

6. Inelastically scattering with chromatic absorption. Due to the chromatic absorption, the visualization properties are similar to those of model 4. However, there are additional visualization possibilities, because the emitted spectrum has spectral components not present in the incident spectrum. Assuming that the materials emit a spectrum with no overlap with the incident spectrum, media may have an absorption value for the incident spectrum that differs from the absorption value for the emitted spectrum. This gives a separate control of the illumination depth and the view depth (Fig. 4bc). The example given in Fig. 4, where the volume image consists of one material, is representative for unsegmented images. Therefore, the availability of different absorption constants provides flexibility in analyzing, in particular, unprocessed images.

Considering the six models, we can draw the following conclusions. Achromatic scattering should not be used as a physical basis for the light/matter interaction model of a volume renderer, because colored absorption, then needed to color an object, does not satisfy the requirement of neutral transmittance. Elastic scattering provides a far better basis because objects can get an individual color. In addition, by using different absorption values for light scattered by different materials, embedded objects can be made visible without disturbing the view on the surface structure of surrounding objects. Inelastic scattering provides an even 


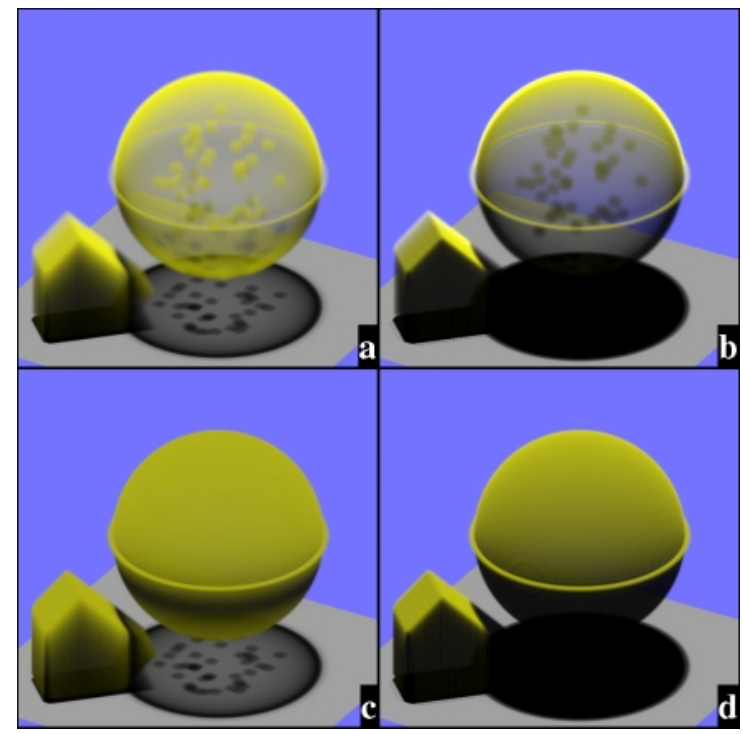

Fig. 4. Visual capabilities of inelastic scattering with chromatic absorption. The emitted light has a longer wavelength than the incident light, therefore the illumination absorption $A_{i l l}$ and radiation absorption $A_{\text {rad }}$ can have different values: a Both $A_{i l l}$ and $A_{\text {rad }}$ have a low value, giving a clear view on the volume structure and shadow. b $A_{i l l} \gg A_{\text {rad }}$, the surface structure is visible through the sharp shadows created in the illumination phase. The volume structure is visible because the view-depth is large, the sphere does not occlude the table. c $A_{\text {ill }} \ll A_{\text {rad }}$, the volume structure is visible through shadows, but also the surface structure through the short view-depth. These absorption settings enable a close look on the light distribution. d Both $A_{i l l}$ and $A_{\text {rad }}$ have a high value, light is almost totally absorbed and only surface structures are visible.

better basis as it also gives the option to use absorption values in the viewing phase that are different from the illumination phase. This creates the possibility to view the illumination distribution (Fig. 4c), or to view the volume and surface structure at the same time (Fig. 4b). From the 6 material models, model 6 , inelastic scattering with chromatic absorption offers the largest visualization flexibility.

\section{Applications}

The following two examples have been reproduced from [8]. We did not reproduce the visualizations of fMRI data. 


\subsection{Vascular Tree}

As a first example (reproduced from [8]), we visualize the veins inside and outside the brain segmented from a MR angiogram recorded with gadolinium (Fig. 5). We visualized the structures as separate entities ( $a$ and b), and combined (c). To see the inner veins we tried a hole (d), transparent brain (e), taking square root of the squared intensities (f), adding (g), or taking a separate channel (h). Our spectral approach is shown in $(\mathrm{i}),(\mathrm{j})$ and $(\mathrm{k})$, the difference between the individual images is that the brain absorbs the light of the inner and background veins more and more.

\subsection{CT: Skin/Bone and Artificial Objects}

Another application of spectral rendering is given in Fig. 6, where the task was to visualize the relation between skin and skull of a patient with Morbus Crouzon. In a normal renderer, it would only be possible to render the skin and skull as separate entities and not in one view (Fig. 6a-c). Spectral rendering gives the option to view the skull inside the skin without degrading the view on the skin (Fig. 6d,e).

Spectral rendering can also be used for interactive analysis. By moving a $3 \mathrm{D}$ cursor which is visible through the skin, but not through the skull, one can interactively estimate the thickness of the skin (Fig. 6f). This is accomplished by letting the $3 \mathrm{D}$ cursor scatter the same spectral band as the skull. Another application of spectral rendering may be to highlight interactive tools, for example, when a $3 \mathrm{D}$ cursor is obscured by surrounding media and the user has lost sight of the cursor.

The bottom row of Fig. 6 shows some visualizations where the restriction of having an absorption value that is constant for both the illumination and the radiation phase has been removed. When there is no absorption during the illumination phase, the skin gets illuminated uniformly (Fig. 6gh). There are no shadows by which every cavity can be inspected in detail. We believe that a slightly less absorption in the illumination phase than in the radiation phase, gives the best compromise between showing all detail and having a good shadow cue (Fig. 6i).

\section{Conclusions}

We proposed a feasible, physical realistic method to visualize volume structures inside other volume structures by modeling the changes in the illumination spectrum when it interacts with the material inside a voxel. Advantages over existing methods are: (1) still getting a visual hint which structures are inside, (2) easy integration with artificial objects, and (3) user selectable shadowing. 


\section{References}

1. J. Burtscher, C.Kremser, M. Seiwald, M. Wagner, F. Aichner, K. Twerdy, and S. Felber, "Three Dimensional Computer Assisted MR-Imaging for Neurosurgical Planning", Proc SPIE 3031, 1997, 85-91. 706

2. J.H. Kim, K.M. Yeon, M.C. Han, D.H. Lee, and H.I. Cho, "Enhanced visualization of MR angiogram with modified MIP and 3D image fusion", Proc SPIE 3031, 1997, 31-36. 706

3. H.T.M. van der Voort, H.J. Noordmans, J.M. Messerli and A.W.M. Smeulders, "Physically realistic volume visualization for interactive analysis", Fourth Eurographics on rendering, 1993, 295-306. 707

4. M. Levoy, "A hybrid ray tracer for rendering polygon and volume data", IEEE Computer Graphics and Applications 10, 1990, 33-40. 708, 709

5. R.A. Drebin, L. Carpenter, P. Hanrahan, "Volume rendering", Computer Graphics (SIGGRAPH '88 Proc) 22(4), 1988, 65-74. 708

6. P. Sabella, "A rendering algorithm for visualizing 3D scalar fields. Computer Graphics (SIGGRAPH '88 Proc) 22(4), 1988, 51-58. 708

7. J.T. Kajiya, and B.P. von Herzen, "Ray tracing volume densities", Computer Graphics (SIGGRAPH '84 Proc) 18(3), 1984, 165-174. 708

8. H.J. Noordmans, H.T.M. van der Voort, G.J.M. Rutten, M.A. Viergever, "Physically realistic visualization of embedded volume structures for medical image data", in SPIE Medical Imaging 1999, Image Display 3658, eds. S.K. Mun, Y. Kim, 1999, 613-620. 712, 713 


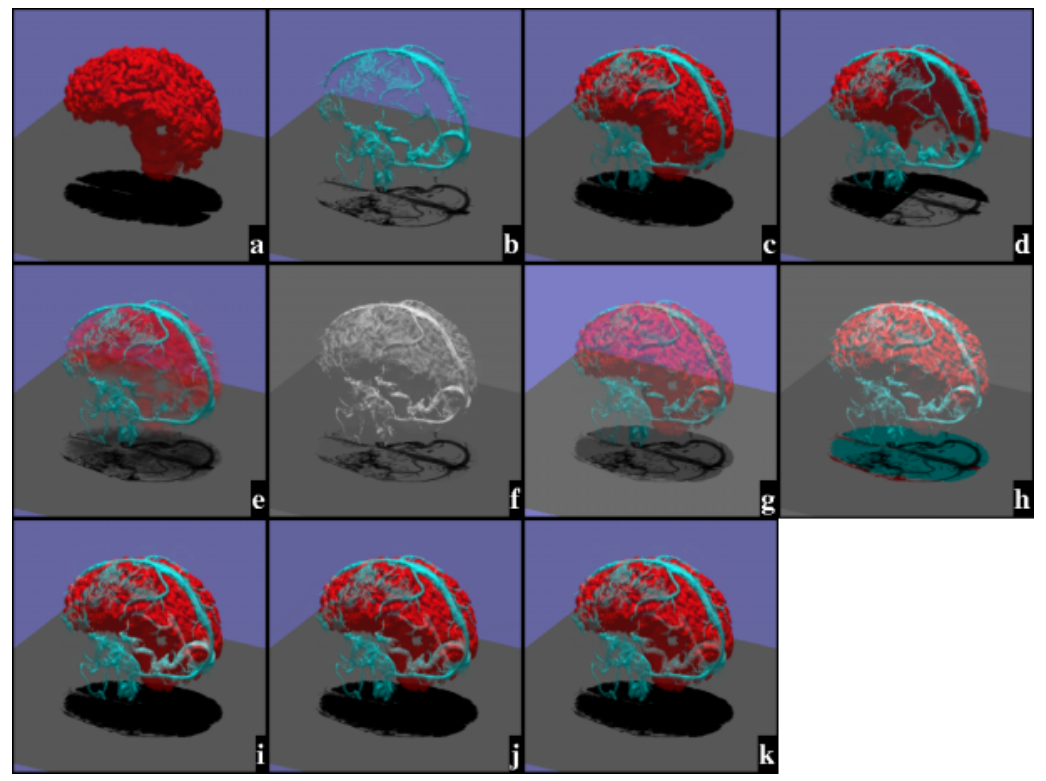

Fig. 5. Options to visualize veins in relation to the brain. (T1 weighted MR scan with gadolinium as contrast agent). a View on the cortex. b View on the veins. c Default way to visualize veins on a cortex. The embedded and background veins are occluded by the brain. d Artificially removing a part of the brain reveals embedded veins, but deteriorates the view on the cortex. e Making the brain transparent reveals embedded veins, but deteriorates the view on the cortex. f Square root squared intensities, grey result. g Averaging RGB values, table and background shine through. h Fig. a placed in red channel, $\mathbf{b}$ in red and blue channel; erroneous colors of table and background. i-k Spectral approach, the brain is transparent for light scattered by the veins, not for light scattered by the brain itself. From $\mathbf{i}$ to $\mathbf{k}$, the brain absorbs the light scattered by the veins more and more. Note that the embedded or 'occluded' veins are white while the unoccluded veins have cyan color. See also http://www.isi.uu.nl/people/herke/neuro/vascular/vascular.html. 


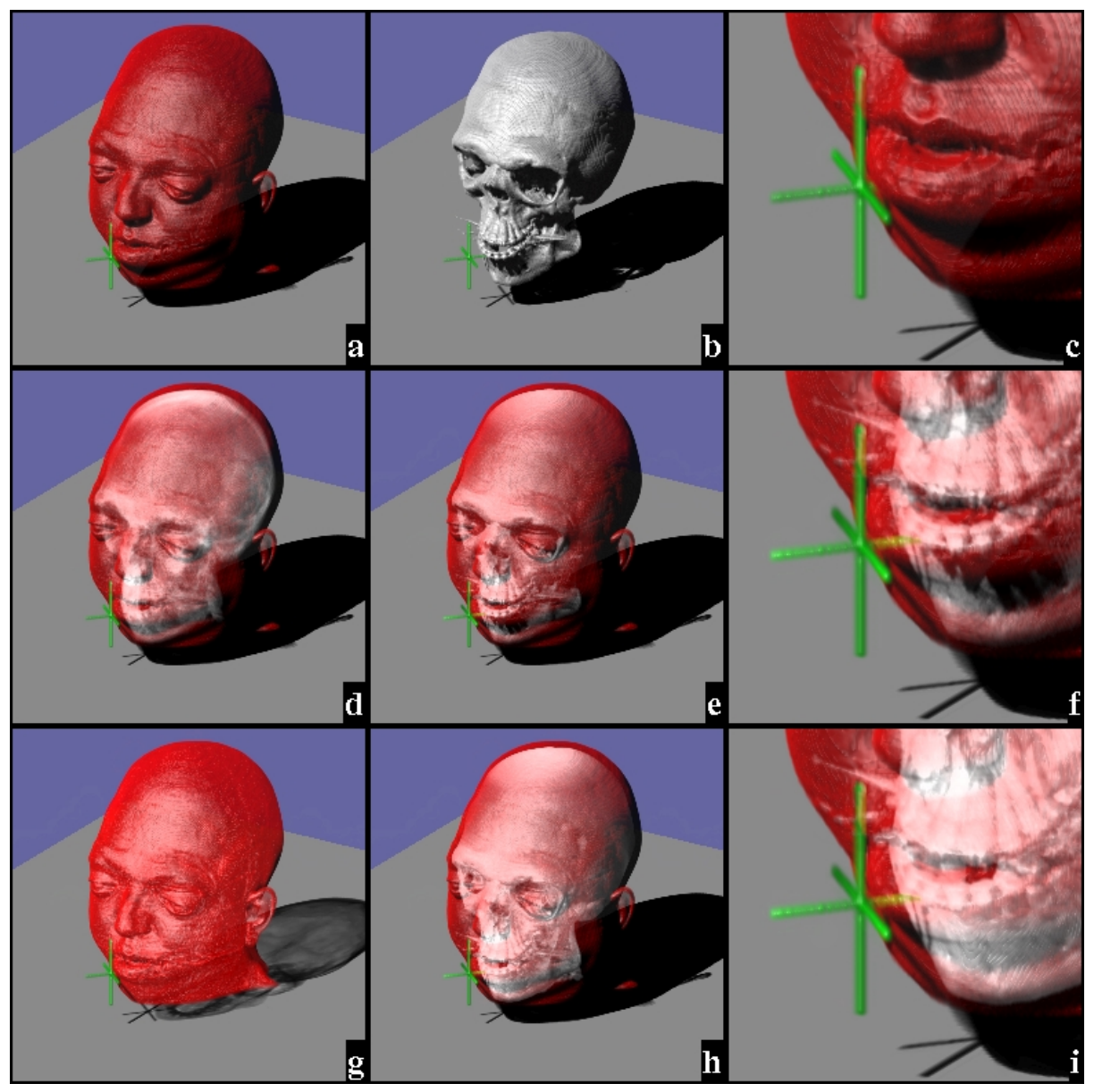

Fig. 6. Volume renderings of a segmented skin and skull from a patient with Morbus Crouzon and a 3D cursor. The image modality is CT, size 320x320x229. The skin, skull scatter different wavelengths: a-c Normal rendering, the absorption is similar for all wavelengths. $\mathbf{d}-\mathbf{i}$ The skin is transparent for light scattered by the skull and 3D cursor. g-i Special scattering process (inelastic): the absorption in the radiation phase differs from the absorption in the illumination phase. Data courtesy University Hospital Utrecht, the Netherlands. See also http://www.isi.uu.nl/people/herke/neuro/ct/ct.html. 